\title{
Caractéristiques physiologiques et survie après plantation de plants de Cedrus atlantica élevés en conteneurs sur différents types de substrats de culture
}

\author{
J.M. Guehl1, G. Falconnet ${ }^{2}$ et J. Gruez² \\ 1 INRA, Laboratoire de bioclimatologie et d'écophysiologie, Station de sylviculture et de production, \\ Centre de Recherches de Nancy, Champenoux, 54280 Seichamps, France;
}

2 CEMAGREF, Division techniques forestières méditerranéennes, Groupement d'Aix-en-Provence, Le Tholonet, 13610 Aix-en-Provence, France

(reçu le 15-3-1988; accepté le 18-8-1988)

Résumé - Des plants de cèdre ont été élevés pendant un an, d'une part en conditions de fertilisation optimale et d'autre part en conditions de fertilisation limitante, en conteneurs sur différents types de substrats de culture. Ces substrats étaient constitués par des mélanges binaires $(50-50 \%$ en volume) et ternaire combinant, d'une part des fibres de bois manufacturées $(\mathrm{H})$ avec de la pouzzolane $(P)$, et d'autre part de la tourbe blonde de sphaigne $(T)$ avec l'un des composants ou mélange suivants : écorce de Pin (E), argile expansée (A), pouzzolane, terre argilo-calcaire (t), mélange d'écorce de pin (40\%) et terre argilo-calcaire (20\%).

La substitution de la tourbe par ces différents composants affecte de façon très sensible les propriétés physico-chimiques du produit résultant. Le substrat apparaît comme un facteur de modulation très important du développement racinaire des plants, de leur croissance, ainsi que de leurs caractéristiques photosynthétiques et transpiratoires déterminées à la fin de la période d'élevage. En conditions de fertilisation optimale, les performances de croissance et de photosynthèse maximales étaient réalisées avec les substrats TA et TE caractérisés par une aération très favorable. En conditions de fertilisation limitante, les performances des substrats comprenant de l'écorce étaient sensiblement plus faibles que celles de TA, probablement en raison d'une carence azotée induite par la poursuite des processus de minéralisation de l'écorce.

Le taux de reprise des plants, transplantés à motte intacte, est lié de façon très étroite à leur capacité photosynthétique totale (surface foliaire $x$ taux d'assimilation de $\mathrm{CO}_{2}$ ) mesurée au moment de la transplantation. Cette relation suggère un lien fonctionnel entre la capacité totale instantanée de production d'assimilats et le potentiel de croissance racinaire et de prospection du sol après transplantation.

Les résultats montrent l'intérêt de l'utilisation de certains substrats de culture artificiels (dans notre cas TA et TE), ayant des propriétés physico-chimiques plus favorables à la croissance des plants que les substrats classiques à base de terre. Ils montrent aussi l'intérêt de pouvoir disposer de critères physiologiques, plus fiables que les critères morphologiques et visuels, pour évaluer la qualité des plants vis-à-vis de la reprise après transplantation. 
Summary - Physiological characteristics and field survival of Cedrus atlantica seedlings grown on different container growth media. Cedrus atlantica seedlings were grown for one year on different container growth media in optimal and limiting fertilization. Different binary $(50-50 \%$ in volume) and ternary substrates were tested, combining manufactured cellulose fibres $(H)$ with pouzzolane $(P)$ and sphagnum peat $(T)$ with one of the following components or mixture : pine bark $(E)$, expanded clay $(A)$, pouzzolane, clayey-calcareous soil (i), pine bark (40\%) and clayeycalcareous soil (20\%).

Varying the second component in these peat containing mixtures affected markedly the relative importance of the air and readily available water volumes.

In optimal nutritional conditions, maximum root development and highest plant growth and $\mathrm{CO}_{2}$ assimilation capacity were obtained with the well-aerated TA and TE substrates. In limiting nutritional conditions, growth and photosynthetic performances of the bark-containing substrates (TE and TEt) were markedly lower than those of the TA mixture, probably owing to a nitrogen deficiency effect due to a significant $\mathrm{N}$ consumption by bark mineralization.

Field survival after transplanting without disturbing the root-substrate systems was closely related to the mean treatment values of whole plant $\mathrm{CO}_{2}$ assimilation capacity (leaf area $\times \mathrm{CO}_{2}$ assimilation rate) measured just before transplanting, which suggests the root growth and soil prospection ability to be related by a functional linkage to the actual photosynthate production capacity.

The results show the advantage of using appropriate artificial growth media (TA and TE in our case) in nursery production rather than soil containing substrates. They also highlight the need for defining physiological criteria - more reliable than morphological and visual criteria - for assessing seedling quality and determining optimal nursery culturing processes.

\title{
Cedrus atlantica - physiological characteristics - field survival - growth media
}

\begin{abstract}
Abréviations : A : taux d'assimilation de $\mathrm{CO}_{2}\left(\mu \mathrm{mol} \mathrm{m} \mathrm{m}^{-2} \mathrm{~s}^{-1}\right)$; $\mathrm{E}$ : taux de transpiration (mmol $\left.\mathrm{m}^{-2} \mathrm{~s}^{-1}\right) ; \mathrm{g}_{\mathrm{s}}$ : conductance stomatique pour la diffusion du $\mathrm{CO}_{2}\left(\mathrm{mmol} \mathrm{m}^{-2} \mathrm{~s}^{-1}\right) ; \mathrm{S}:$ surface foliaire totale par plant; $\mathrm{SA}$ : capacité totale d'assimilation de $\mathrm{CO}_{2}$ par plant. $\mathrm{HP}$ : mélange fibres de bois manufacturées et pouzzolane (50-50\% en volume); TA : mélange tourbe de sphaigne et argile expansée (50-50\%); TE : mélange tourbe et écorce de pin (50-50\%); TEt : mélange tourbe, écorce et terre argilo-calcaire (40-40-20\%); TP : mélange tourbe et pouzzolane (50-50\%); Tt : mélange tourbe et terre argilo-calcaire (50-50\%).
\end{abstract}

\section{Introduction}

Depuis une vingtaine d'années, l'utilisation de plants forestiers résineux en conteneurs s'est particulièrement développée en zone méditerranéenne et porte actuellement sur 3 à 5 millions d'unités par année. Cependant, la mortalité après plantation sur le terrain reste élevée, nécessitant de coûteuses opérations d'entretien et de regarnis. Par ailleurs, les lots de plants produits à l'aide des méthodes traditionnelles se caractérisent souvent par l'existence d'une très forte hétérogé- néité de taille des plants rendant leur gestion problématique.

Face à ces constats, l'effort d'optimisation des procédés de culture en pépinière forestière reste insuffisant, et les connaissances actuelles ne permettent pas de faire des choix qui satisfassent à la fois le pépiniériste et le reboiseur. Le support de culture est un facteur de modulation important de l'environnement physico-chimique racinaire, pouvant créer des conditions plus ou moins favorables au développement et à la nutrition des plants (André, 1987; Gras, 1987). Les effets de 
substrats de culture sur la croissance ont été examinés chez plusieurs ligneux ornementaux (Lemaire et al., 1980). Pour les plants forestiers, en dehors de résultats obtenus sur plusieurs feuillus (Garbaye et Le Tacon, 1978; Riedacker, 1978a; Garbaye, 1986), les gains de croissance susceptibles d'être obtenus en pépinière par optimisation du substrat et de la fertilisation restent largement inconnus. D'autre part, dans le domaine forestier, les critères de qualité des plants doivent également prendre en compte, même pour les plants élevés en conteneurs, les contraintes liées à la transplantation et l'adaptation aux conditions souvent défavorables du site d'accueil. Et il n'est pas certain que les substrats permettant une croissance maximale en pépinière soient également ceux entraînant l'optimisation des performances après plantation.

Pour apporter des réponses à ces questions, la Division Techniques Forestières Méditerranéennes du CEMAGREF met en cuuve depuis 1985 un programme d'expérimentation en vraie grandeur reposant sur l'utilisation de plants de cèdre cultivés sur différents types de substrats. Dans l'objectif de mieux comprendre les résultats obtenus, nous avons procédé, à la fin de la période d'élevage des plants, à l'examen des caractéristiques morphologiques des plants, notamment au niveau racinaire, et de leurs caractéristiques photosynthétiques et transpiratoires.

\section{Matériels et Méthodes}

\section{Production des plants}

Des graines de Cedrus atlantica récoltées dans le sud de la France (Ventoux pour les plants produits en 1985, et Luberon pour les plants produits en 1986) ont été semées en avril 1985 puis en avril 1986 dans la pépinière des Milles, près d'Aix-en-Provence (Direction Départementale de l'Agriculture des Bouches-du-Rhône) sur différents substrats artificiels remplissant des conteneurs antispiralisation (Riedacker, 1978b) de $17 \mathrm{~cm}$ de haut et $400 \mathrm{~cm}^{3}$ de volume et placés sous une ombrière interceptant $40 \%$ du rayonnement global incident.

Pour l'expérimentation de 1985, 13 types de substrats binaires $(50 \%$ en volume pour chacun des constituants) ont été utilisés dont 4 ont été retenus pour la présente étude :

- TE : tourbe blonde et écorce de pin maritime compostée;

- TA : tourbe et argile expansée (diamètre des éléments $1,2 \mathrm{~cm}$ );

- TP : tourbe el pouzzolane (origine Agde, Hérault);

- HP : fibres cellulosiques manufacturées (Hortifibre) et pouzzolane.

Dans ces différents mélanges, la tourbe et dans une moindre mesure l'écorce interviennent pour leurs caractéristiques intéressantes de réservoir d'eau (Gras, 1987) et les autres constituants pour le maintien d'une macroporosité élevée favorisant l'aération.

La culture a été conduite de façon à maintenir, grâce à un système de pesée des plants et à des apports d'eau journaliers, les disponibilités hydriques dans le substrat à un niveau non limitant pour les plants $(\mathrm{pF}<2)$. II est toutefois possible que, pour HP, ce niveau de sécheresse ait été dépassé lors des situations de forte demande évaporative, en raison des caractéristiques hydriques défavorables de ce substrat. Le niveau nutritionnel des substrats a été maintenu à un niveau supposé être optimal, grâce à des apports de solution nutritive de type CoicLesaint (formule 12-8-30) utilisée à la concentration de $200 \mathrm{gl}^{-1}$ et diluée à $5 \%$ dans l'eau d'arrosage (arrosage fertilisant une fois par semaine en moyenne). Le contrôle du niveau nutritionnel de la solution du substrat était effectué globalement par la mesure du $\mathrm{pH}$ et de la conductivité électrique. Les apports de solution nutritive ont été interrompus après l'arrêt de la croissance en hauteur des plants au 15 septembre.

Pour l'expérimentation de 1986, des modalités expérimentales différentes ont été mises en œuvre. Outre les substrats TE (écorce de pin Laricio en 1986), TA et TP déjà utilisés en 1985, on a étudié les mélanges de composition suivants :

- $\mathrm{Tt}$ : tourbe blonde et terre argilo-calcaire;

- TEt : tourbe $(40 \%)$, écorce de pin Laricio $(40 \%)$ et terre argilo-calcaire $(20 \%)$. 
De tels mélanges, comprenant de la terre, sont fréquemment utilisés par les pépiniéristes. De plus, en 1986 les apports d'éléments nutritifs ont été diminués de moitié par rapport à 1985 (concentration de la solution initiale réduite à $100 \mathrm{gl}^{-1}$ ) de façon à étudier l'effet du substrat en conditions nutritionnelles limitantes. La fraction volumique relative des phases gazeuse, liquide et solide des substrats utilisés en 1986 (hormis HP), ainsi que la proportion d'eau disponible entre pF 1 et pF 2 ont été déterminées au Laboratoire de Chimie et Sciences du sol de l'ENITH à Angers (Lemaire et al., 1980). Ces caractéristiques sont indiquées dans la Figure 1.

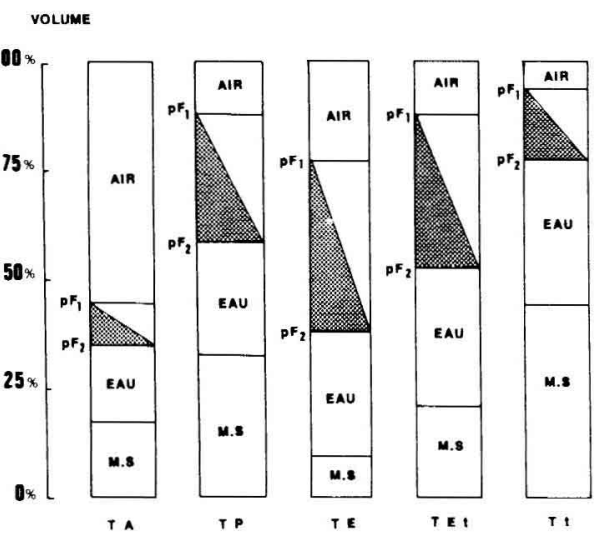

Fig. 1. Fraction volumique des phases gazeuse, liquide et solide et importance de la réserve hydrique disponible entre $\mathrm{pF} 1$ et $\mathrm{pF} 2$ pour les différents substrats.

\section{Installation des boisements expérimen- taux}

Les plants produits en 1985 ont été transplantés sur le terrain en février 1986 sur le site de Montaren, près d'Uzès, dans le département du Gard. Les plants de 1986 ont été transplantés en novembre et décembre 1986 en 3 sites caractéristiques de la limite inférieure de l'aire d'introduction du cèdre en zone méditerranéenne (étage méditerranéen inférieur, série du chêne vert) : Montaren, Poulx (Gard) et les Baux-de-Provence (Bouches-du-Rhône). Pour chaque type de substrat et site, 90 plants ont été installés. Simultanément à l'installation des boisements expérimentaux, des plants ont été utilisés pour l'établissement de leurs caractéris- tiques morphologiques, de biomasse et de fonctionnement photosynthétique et transpiratoire.

\section{Observations racinaires}

Les points suivants ont été relevés :

- appréciation qualitative de la densité du chevelu racinaire (racines en croissance et racines ayant cessé leur croissance);

- ordre maximum de ramification à partir du pivot (ordre 1), uniquement en 1986;

- nombre de racines en cours d'élongation (présentant des extrémités blanches);

- appréciation qualitative de l'abondance du système de racines latérales courtes;

- existence de déformations racinaires, notamment au niveau des racines primaires.

Mesure des échanges gazeux de $\mathrm{CO}_{2}$ et $\mathrm{H}_{2} \mathrm{O}$

Les mesures ont été réalisées à l'aide d'un système ouvert comprenant 3 chambres d'assimilation placées en parallèle et régulées en température (température de l'air maintenue à $22^{\circ} \mathrm{C}$ durant les mesures) ainsi que pour l'humidité et la concentration en $\mathrm{CO}_{2}$ à l'entrée des chambres. La concentration en $\mathrm{CO}_{2}$ à l'entrée des chambres était mesurée en permanence à l'aide d'un analyseur par absorption infrarouge de type ADC-225 MK2 et maintenue à $330 \pm 5$ $\mathrm{Pa} \mathrm{MPa}^{-1}$. La différence de concentration en $\mathrm{CO}_{2}$ entre l'entrée et la sortie des chambres était mesurée à l'aide d'un analyseur différentiel de type ADC-2.25 MK3 alternativement pour les 3 chambres grâce à un système d'électrovannes commandées automatiquement (séquences de mesures de 2 à 5 min par position). Le point de rosée de l'air entrant dans les chambres puis de l'air sortant des chambres était mesuré avec un hygromètre à point de rosée (System 1100 DP de General Eastern) par séquences simultanées à celles des mesures de $\mathrm{CO}_{2}$. La différence de pression partielle de $\mathrm{H}_{2} \mathrm{O}$ entre feuille et atmosphère était égale à $8 \pm 1 \mathrm{~Pa} \mathrm{KPa}^{-1}$. La densité du flux de photons photosynthétiquement actifs (lampe à vapeur de sodium haute pression) était de $600 \mu \mathrm{mol} \mathrm{m} \mathrm{m}^{-2} \mathrm{~s}^{-1}$. Pour la détermination du taux d'assimilation de $\mathrm{CO}_{2}$ (A) et de transpiration $(E)$, on a rapporté les mesures à la surface extérieure totale des aiguilles déterminée sur la base de leur surface projetée à l'aide d'un analyseur d'images. Les aiguilles ont été assimi- 
Tableau I. Dimensions et poids de matière sèche de plants de cèdre âgés de un an élevés en 1985 (fertilisation optimale) et en 1986 (fertilisation limitante) sur différents types de substrats de culture. Pour chaque colonne, des valeurs moyennes par traitements suivies de lettres toutes différentes indiquent une différence au seuil de $5 \%$ pour le test de comparaison multiple de Scheffe.

\begin{tabular}{|c|c|c|c|c|c|c|}
\hline $\begin{array}{l}\text { Substrat et } \\
\text { nombre } \\
\text { de répétitions }\end{array}$ & $\begin{array}{l}\text { Hauteur } \\
\text { des plants } \\
(\mathrm{mm})\end{array}$ & $\begin{array}{c}\text { Diamètre } \\
\text { au collet } \\
\text { (mm) }\end{array}$ & $\begin{array}{c}\text { Poids racines } \\
\text { (g) }\end{array}$ & $\begin{array}{l}\text { Poids partie } \\
\text { aérienne } \\
\text { (g) }\end{array}$ & $\begin{array}{l}\text { Poids total } \\
\text { (g) }\end{array}$ & $\begin{array}{l}\text { Poids racines } \\
\text { Poids total }\end{array}$ \\
\hline \multicolumn{7}{|l|}{1985} \\
\hline $\mathrm{HP}(6)$ & $91 \mathrm{bc}$ & $2,1 \mathrm{c}$ & $0,37 d$ & $0,46 \mathrm{c}$ & $0,83 \mathrm{c}$ & 0,45 bcde \\
\hline TP (9) & $162 \mathrm{a}$ & $3,3 a b$ & $1,08 \mathrm{ab}$ & $1,59 \mathrm{ab}$ & $2,67 \mathrm{a}$ & $0,40 \mathrm{e}$ \\
\hline TA (9) & $158 a$ & $3,7 \mathrm{a}$ & $1,23 a b$ & $1,74 a b$ & $2,97 \mathrm{a}$ & $0,41 \mathrm{de}$ \\
\hline TE (9) & $194 \mathrm{a}$ & $3,8 \mathrm{a}$ & $1,32 \mathrm{a}$ & $1,99 \mathrm{a}$ & $3,31 \mathrm{a}$ & 0,40 de \\
\hline \multicolumn{7}{|l|}{1986} \\
\hline TEt (12) & $65 c$ & $2,2 \mathrm{c}$ & $0,45 d$ & $0,35 \mathrm{c}$ & $0,80 \mathrm{c}$ & $0,56 \mathrm{a}$ \\
\hline $\mathrm{Tt}(12)$ & $108 \mathrm{~b}$ & $2,5 \mathrm{c}$ & $0,64 \mathrm{~cd}$ & $0,64 \mathrm{c}$ & $1,28 \mathrm{bc}$ & $0,50 \mathrm{abc}$ \\
\hline $\mathrm{TP}(12)$ & $102 b$ & $3,2 a b$ & $0,89 \mathrm{bc}$ & $0,82 \mathrm{c}$ & $1,71 \mathrm{~b}$ & $0,52 a b$ \\
\hline TA (12) & $159 a$ & $3,2 a b$ & $1,13 a b$ & $1,42 b$ & $2,55 \mathrm{a}$ & 0,44 cde \\
\hline TE (12) & $109 \mathrm{~b}$ & $2,8 \mathrm{bc}$ & $0,59 \mathrm{~cd}$ & $0,65 \mathrm{c}$ & $1,24 \mathrm{bc}$ & $0,48 \mathrm{bcd}$ \\
\hline
\end{tabular}

Tableau II. Morphologie racinaire de plants de cèdre âgés de un an élevés en 1985 (fertilisation optimale) et en 1986 (fertilisation limitante) sur différents types de substrat de culture.

\begin{tabular}{|c|c|c|c|c|}
\hline $\begin{array}{l}\text { Substrat et } \\
\text { nombre de répétitions }\end{array}$ & $\begin{array}{l}\text { Nombre d'extrémités } \\
\text { blanches }\end{array}$ & $\begin{array}{l}\text { Densité de racines } \\
\text { latérales courtes }\end{array}$ & $\begin{array}{l}\text { Densité du } \\
\text { chevelu racinaire }\end{array}$ & $\begin{array}{l}\text { Ordre maximal } \\
\text { de ramifications }\end{array}$ \\
\hline \multicolumn{5}{|l|}{1985} \\
\hline $\mathrm{HP}(6)$ & $1,5 b$ & très faible & très faible & - \\
\hline TP (9) & $11 \mathrm{a}$ & moyenne & faible & - \\
\hline TA (9) & $9,5 \mathrm{a}$ & forte & forte & - \\
\hline TE (9) & $14,2 \mathrm{a}$ & moyenne & forte & - \\
\hline \multicolumn{5}{|l|}{1986} \\
\hline TEt (12) & $10 a$ & faible & très faible & 3 \\
\hline$T t(12)$ & $8 \mathrm{a}$ & faible & très faible & 3 \\
\hline$T P(12)$ & 9,4 a & moyenne & moyenne & 3 \\
\hline TA (12) & $18,2 \mathrm{a}$ & forte & forte & 4 \\
\hline TE (12) & $13 a$ & moyenne & forte & 3 \\
\hline
\end{tabular}

lées à des cylindres. Pour les calculs de $A, E$ et de la conductance stomatique pour la diffusion du $\mathrm{CO}_{2}\left(\mathrm{~g}_{\mathrm{s}}\right)$, on a utilisé les équations classiques données par Caemmerer et Farquhar (1981).

\section{Résultats}

Caractéristiques dimensionnelles, pondérales et morphologiques des plants

Les données des Tableaux I et II font apparaître, pour les 2 années d'étude, un effet important du substrat sur les dimensions et le poids de matière sèche des plants ainsi que leur morphologie racinaire.

En 1985, les plus fortes performances étaient atteintes par le substrat TE. TA et TP se caractérisaient par des valeurs légèrement plus faibles, mais les différences entre TE, TA et TP n'étaient pas statistiquement significatives. Sur le substrat HP, la croissance des plants était extrêmement faible, n'atteignant pour les critères pondéraux qu'environ $25 \%$ des 
valeurs de TE. L'importance pondérale relative des racines était très voisine de 0,40 pour TE, TA et TP et atteignait 0,45 pour HP.

Les 2 substrats contenant de la pouzzolane se caractérisaient par une faible densité du chevelu racinaire. Pour TP, le nombre de racines en élongation (présentant des extrémités blanches) était toutefois du même ordre de grandeur que pour TE et TA, mais la longueur des extrémités blanches était plus courte. Dans le cas du traitement HP, le nombre de racines en élongation était extrêmement faible (2 en moyenne). La densité de racines latérales courtes était très élevée dans le cas du traitement TA. Cette densité était sensiblement plus faible pour TE et TP, et surtout pour HP.

Les traitements contenant de la pouzzolane se caractérisaient par l'existence de déformations racinaires à angle droit ou à $180^{\circ}$ (étranglements), affectant notamment les racines primaires. L'origine de ces déformations semble être due à un piégeage des apex racinaires par la porosité de surface, de 1 à $2 \mathrm{~mm}$ de diamètre, des éléments de pouzzolane. Un tel piégeage peut entraîner soit l'arrêt de croissance de l'apex avec ou sans régénération racinaire en amont (déformations à angle droit ou arrêt total de la croissance), soit une inversion transitoire de la direction de croissance racinaire (étranglements à $180^{\circ}$ ).

En 1986, la réduction de la fertilisation par rapport à 1985 a eu pour effet une réduction de la taille et du poids des plants TE, TA et TP. Les plus fortes valeurs étaient notées pour TA. Contrairement à 1985, le traitement TE se caractérisait en 1986 par des valeurs inférieures à celles de TA et également, quoique de façon non significative, à celles de TP. Les performances de $\mathrm{Tt}$ étaient très voisines de celles de TE. L'introduction d'écorce dans le mélange tourbe-terre (traitement TEt) a réduit de façon systématique les différents paramètres de taille et de poids sans pour autant que les différences soient toujours significatives entre $\mathrm{TEt}, \mathrm{Tt}$ et TE. II est à remarquer que, en dehors de TA, l'importance pondérale relative des racines était plus élevée en 1986 (entre

Tableau III. Caractéristiques photosynthétiques et rapport $\mathrm{g}_{\mathrm{s}} / \mathrm{A}$ avant plantation de plants de cèdre de un an élevés en 1985 (fertilisation optimale) et en 1986 (fertilisation limitante) en conteneurs sur différents types de substrats, et taux de survie des plants en juillet et septembre 1986 (plants élevés en 1985 et transplantés en novembre et décembre 1985) et en septembre 1987 (plants élevés en 1986 et transplantés en février 1987).

\begin{tabular}{|c|c|c|c|c|c|c|}
\hline $\begin{array}{l}\text { Substrat et } \\
\text { nombre de } \\
\text { répétitions }\end{array}$ & $\begin{array}{l}\text { Surface foliaire } \\
\left(S, 10^{-2} \mathrm{~m}^{2}\right)\end{array}$ & $\begin{array}{l}\text { Taux d'assimilation } \\
\text { de } \mathrm{CO}_{2} \\
\left(\mathrm{~A}, \mu \mathrm{mol} \mathrm{m}^{-2} \mathrm{~S}^{-1}\right)\end{array}$ & $\begin{array}{l}\text { Conductance stomatique' } \\
\text { Taux d'as. de } \mathrm{CO}_{2} \\
\left(g_{s} / \mathrm{A}, 10^{3} \mathrm{~mol} \mathrm{~mol}^{-1}\right)\end{array}$ & $\begin{array}{l}\text { Capacité totale } \\
\text { dassimilation de } \mathrm{CO}_{2} \\
\left(\mathrm{SA}, 10^{-2} \mu \mathrm{mol} \mathrm{s}^{-1}\right)\end{array}$ & Taux de & e survie \\
\hline \multicolumn{5}{|l|}{1985} & 07.86 & 09.86 \\
\hline HP (6) & $0,48 d$ & $1,81 \mathrm{bcd}$ & $6,5 \mathrm{~b}$ & $0,87 \mathrm{e}$ & 0,18 & 0,06 \\
\hline TP (9) & $1,62 a b$ & $2,44 a b$ & $6,8 \mathrm{~b}$ & $3,95 b c$ & 0,58 & 0,31 \\
\hline TA (9) & $1,56 a b c$ & $3,01 \mathrm{a}$ & $6,9 \mathrm{~b}$ & $4,70 a b$ & 0,76 & 0,43 \\
\hline TE (9) & $1,86 \mathrm{a}$ & $3,03 a$ & $6,7 \mathrm{~b}$ & $5,64 \mathrm{a}$ & 0,83 & 0,61 \\
\hline \multicolumn{6}{|l|}{1986} & 09.87 \\
\hline TEt (12) & $0,53 d$ & $1,24 \mathrm{~d}$ & $8,2 a b$ & $0,66 \mathrm{e}$ & - & 0,86 \\
\hline $\mathrm{Tt}(12)$ & $0,91 \mathrm{~cd}$ & $1,69 \mathrm{bcd}$ & $7,2 a b$ & $1,54 \mathrm{de}$ & - & 0,93 \\
\hline $\mathrm{TP}(12)$ & $1,05 \mathrm{bcd}$ & 1,77 bcd & $6,9 \mathrm{~b}$ & 1,86 de & - & 0,95 \\
\hline TA (12) & $1,83 a$ & $1,50 \mathrm{~cd}$ & $9 a$ & $2,74 \mathrm{~cd}$ & - & 0,98 \\
\hline TE (12) & $0,79 d$ & $2,19 a b c$ & $6,5 \mathrm{~b}$ & 1,73 de & - & 0,94 \\
\hline
\end{tabular}


$0,44$ et 0,56$)$ qu'en $1985(0,40$ à 0,45$)$. Pour les 2 années d'étude, le poids relatif des racines était le plus élevé pour les traitements (HP puis TEt) ayant produit les plants les plus petits. En 1986, le nombre d'extrémités blanches en croissance et leur longueur étaient les plus élevées pour les substrats TA et TE présentant la meilleure aération. Pour les substrats comprenant de la terre (Tt et TEt), ces extrémités blanches étaient surtout localisées à l'interface substrat-conteneur. Cette observation est à mettre en relation avec la très faible aération des mélanges comprenant de la terre. L'interface substrat-conteneur constitue en effet une zone de transfert d'oxygène favorable au développement racinaire. La densité des racines latérales était, en 1986, très forte pour TA et était sensiblement plus faible pour les autres traitements. De plus, les plants du traitement TA se caractérisaient par l'existence de nombreuses racines d'ordre 4, cependant que pour les autres traitements les racines d'ordre 4 étaient extrêmement rares.

\section{Capacité photosynthétique des plants}

Les caractéristiques photosynthétiques des plants sont fortement modulées par le type de substrat (Tableau III). Cet effet porte à la fois sur la surface foliaire (S) des plants et sur la densité de flux d'assimilation de $\mathrm{CO}_{2}$ (A). II n'y a de relation évidente entre $S$ et $A$ ni pour ce qui concerne la variabilité individuelle globale des plants (Fig. 2), ni pour les valeurs moyennes par traitement de ces 2 composantes de la capacité totale d'assimilation des plants. En 1985, les valeurs maximales de $A$ étaient atteintes pour TE et TA (Tableau III). La surface foliaire des plants TA était en moyenne inférieure, mais de façon non significative, à celle des plants TE. Les plants TP avaient une surface voisine de celle des plants TA, mais les valeurs de $A$ et de $S A$ étaient plus faibles (significativement si les tests statistiques sont effectués pour la seule année 1985) pour TP. Les très faibles valeurs de capacité photosynthétique totale des plants HP sont dues à des faibles valeurs de $\mathrm{A}$ et surtout de S.

Pour les traitements TE et TP, les valeurs de $A$ et de $S A$ étaient inférieures, en 1986, à celles de 1985. Pour TA, ce résultat est également observé concernant $A$ et $S A$, mais non pour $S$. En 1986, la plus forte valeur de $S A$ était observée pour TA; les valeurs moyennes des traitements TE, TP et Tt sont nettement inférieures à celles de TA, mais les différences entre traitements ne sont pas significatives en raison de la forte variabilité individuelle intra-traitement.

Les données des Tableaux II et III font apparaître une nette relation entre les valeurs moyennes de SA et du poids total des plants. On a représenté sur la Figure 3a cette même relation obtenue pour les valeurs individuelles de SA et de poids de matière sèche. Cette relation se caractérise par une absence de linéarité et une dispersion des points relativement importante, notamment liée à la non-superposition des relations de 1985 et de 1986. La relation globale entre poids de matière sèche et SA est uniquement liée à celle existant entre poids de matière sèche et $S$ (Fig. 3b); le poids total des plants et $A$ sont totalement indépendants (Fig. 3c).

\section{Relation entre assimilation de $\mathrm{CO}_{2}$ et conductance stomatique}

La relation entre $A$ et $g_{s}$ obtenue avec l'ensemble des valeurs individuelles des plants présente globalement une allure linéaire et passe par l'origine (Fig. 4). On notera que les points de 1985 se caractérisent par une relation linéaire très étroite occupant la partie supérieure de la relation globale. Cette observation traduit le fait que les plants élevés en 1986 ont globale- 


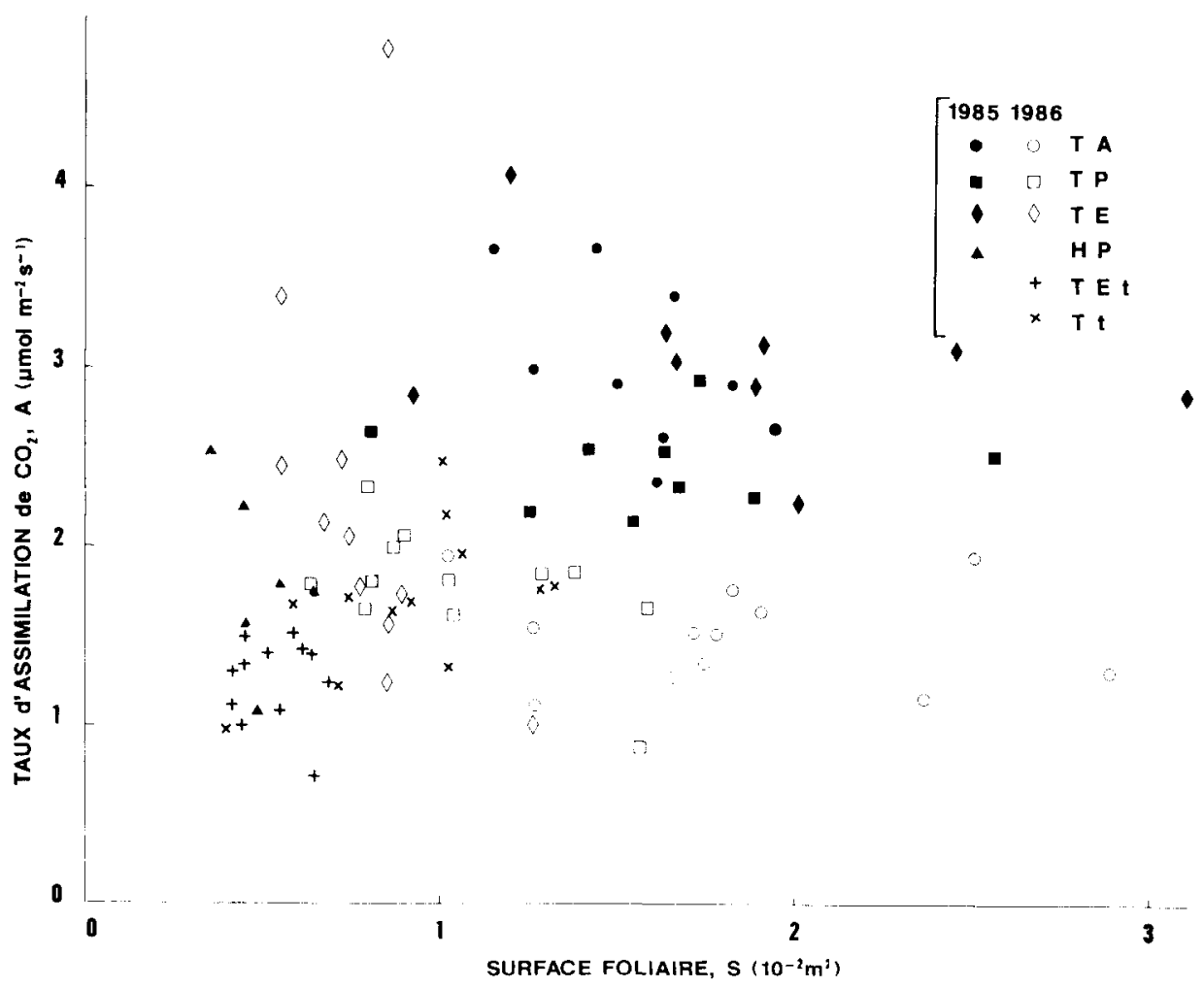

Fig. 2. Taux d'assimilation de $\mathrm{CO}_{2}$ (A) en fonction de la surface foliaire (S) de plants de cèdre élevés en conteneurs sur différents types de substrats de culture en 1985 (fertilisation optimale) et en 1986 (fertilisation limitante).

ment un coût en eau pour l'assimilation de $\mathrm{CO}_{2}$ plus élevé qu'en 1985. Les valeurs de $g_{s} / A$ rapportées par le Tableau III confirment bien ce résultat et font apparaître un net effet du type de substrat pour les plants de 1986, avec des valeurs très élevées pour TA et TEt. En 1985, en revanche, ce rapport est très stable entre les traitements.

\section{Reprise des plants après transplantation}

Les taux de survie ont été déterminés en juillet puis en septembre 1986 pour les plants élevés en 1985, et en septembre 1987 pour les plants élevés en 1986
(Tableau III). En 1986, année climatiquement caractérisée par un déficit hydrique estival très prononcé $(52 \mathrm{~mm}$ de précipitations du fer mai au 31 août à Uzès, soit un déficit de $152 \mathrm{~mm}$ par rapport à la normale), les taux de survie étaient très faibles par rapport à 1987, année climatiquement très favorable à la reprise (238 $\mathrm{mm}$ entre le 1er mai et le 31 août, soit un excédent de $40 \mathrm{~mm}$ par rapport à la normale). Pour chacune des 2 années d'observation, les taux de survie (Tableau III) sont très nettement et positivement corrélés avec les valeurs moyennes par traitement du poids de matière sèche des plants (Tableau I), et sont également les 

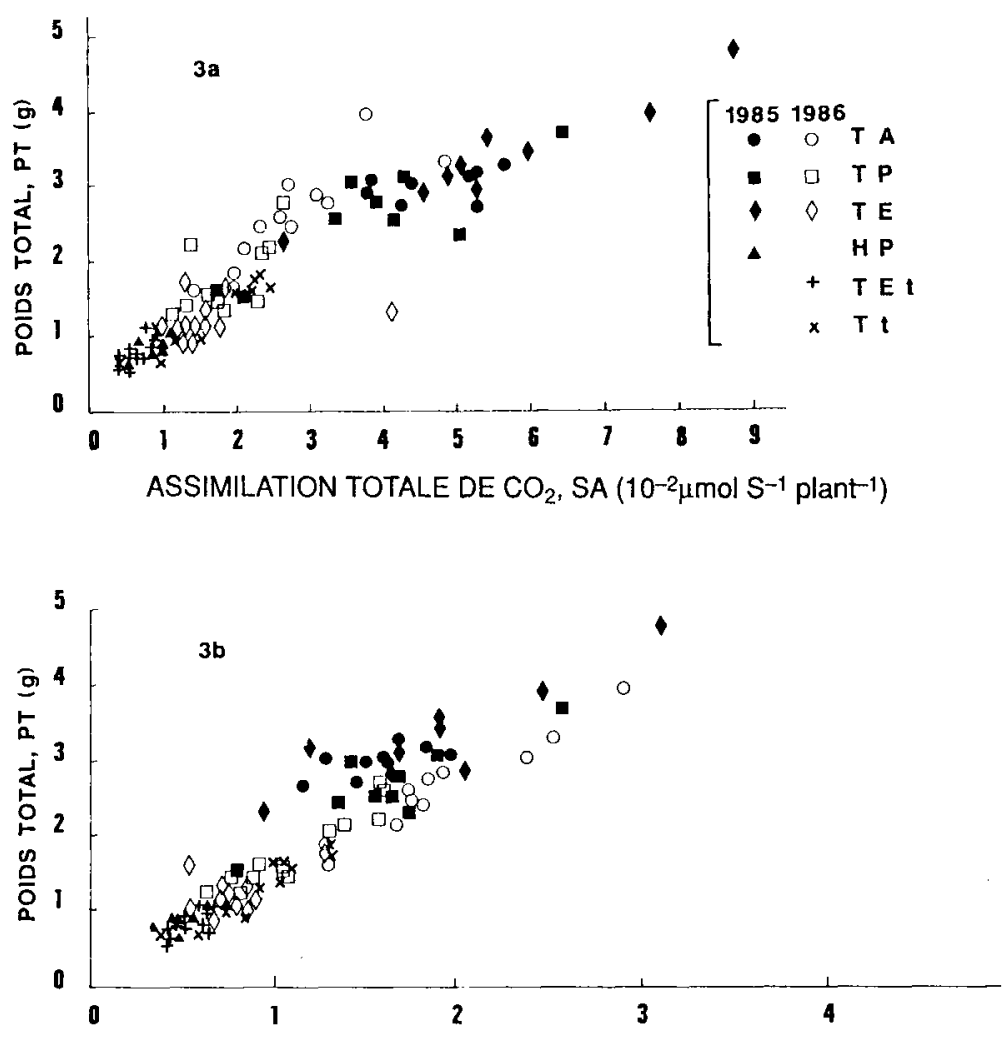

b

SURFACE FOLIAIRE, $\mathrm{S}\left(10^{-2} \mathrm{~m}^{-2}\right)$

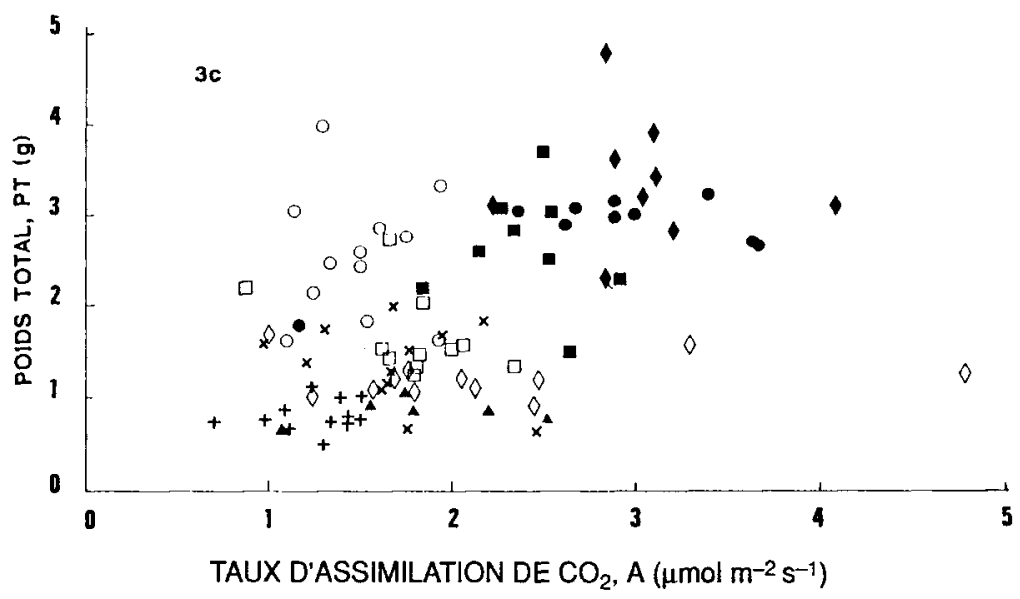

Fig. 3. a, b et c. Poids total de matière sèche (PT) en fonction (a) de la capacité d'assimilation totale des plants (SA). (b) de leur surface foliaire (S) et (c) du taux d'assimilation de $\mathrm{CO}_{2}$ (A) de plants de cèdre élevés sur différents types de substrats de culture en 1985 (fertilisation optimale) et en 1986 (fertilisation limitante). 


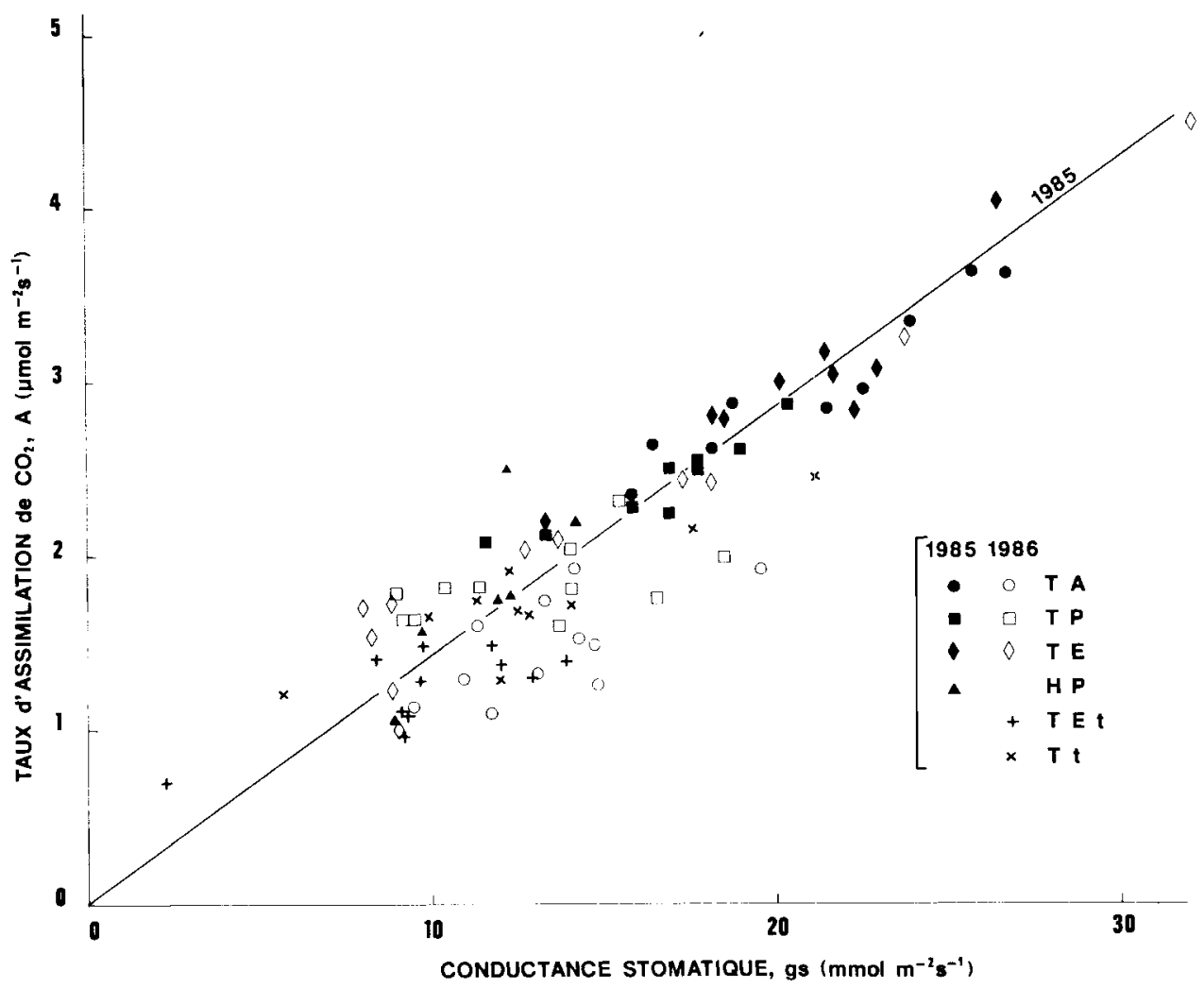

Fig. 4. Taux d'assimilation de $\mathrm{CO}_{2}(\mathrm{~A})$ en relation avec la conductance stomatique $\left(\mathrm{g}_{\mathrm{s}}\right)$ de plants de cèdre élevés sur differents types de substrats de culture avec une fertilisation optimale (1985) et une fertilisation limitante (1986).

plus importantes pour les traitements présentant les plus fortes activités racinaires (Tableau II). II n'est toutefois pas permis de déduire les liens de cause à effet de ces corrélations. L'examen des relations entre taux de survie et les caractéristiques photosynthétiques des plants (Tableau II et Fig. 5) permet de dégager des éléments d'analyse plus précis. Pour chacune des 2 années d'observation, on note une relation positive entre taux de survie et surface foliaire des plants (Fig. 5a). Des corrélations similaires, non montrées ici, existent entre taux de survie et certains paramètres morphologiques tels le diamètre au collet des plants ou leur poids total. Des relations positives sont également observées entre taux de survie et $\mathbf{A}$ (Fig. 5b). La qualité des relations est cependant très nettement améliorée par la combinaison des aspects extensifs $(S)$ et intensif (A) de la capacité photosynthétique totale des plants (Fig. $5 \mathrm{c}$ ).

\section{Discussion}

Le changement des composants associés à la tourbe dans les différents mélanges binaires ou ternaires utilisés dans la pré- 
SEPT. 1987

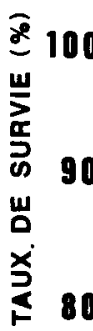

a

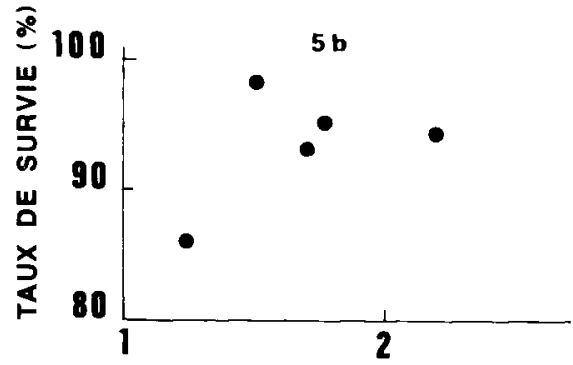

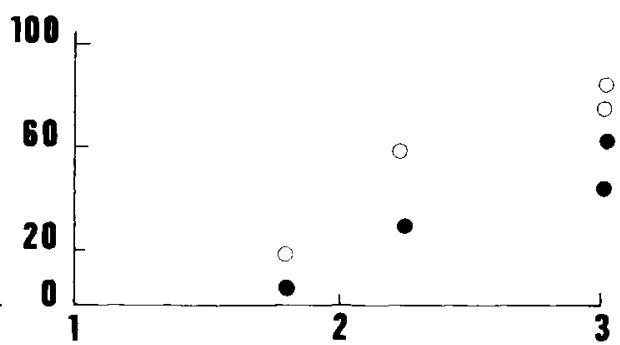

b

TAUX d'ASSIMILATION TOTALE DE $\mathrm{CO}_{2}, \mathrm{~A}\left(\mu \mathrm{mol} \mathrm{m} \mathrm{m}^{-2} \mathrm{~s}^{-1}\right)$

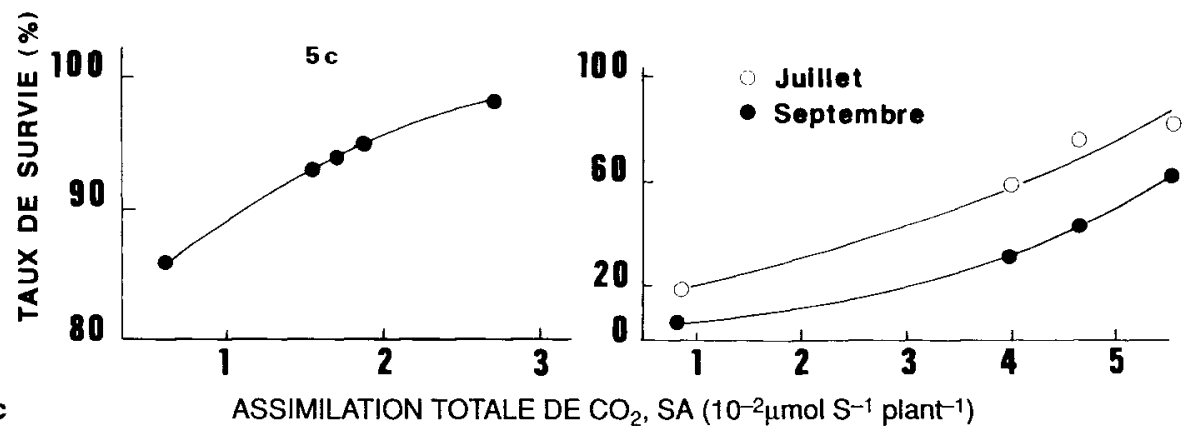

Fig. 5. a, b et c. Taux de survie de plants de cèdre élevés sur différents types de substrats de culture en relation avec les valeurs moyennes par traitement (a) de la surface foliaire des plants (S), (b) du taux d'assimilation de $\mathrm{CO}_{2}$ (A) et (c) de la capacité d'assimilation de $\mathrm{CO}_{2}$ totale des plants (SA). Les caractéristiques d'assimilation de $\mathrm{CO}_{2}$ des plants ont été établies avant la transplantation. A droite, survie en juillet puis septembre 1986 des plants élevés en 1985 (fertilisation optimale) et plantés en novembre et décembre 1985. A gauche, survie en septembre 1987 des plants élevés en 1986 (fertilisation limitante) et plantés en février 1987.

sente étude induit des différences très marquées dans les caractéristiques physiques résultantes des substrats (Fig. 1). La variabilité de ces caractéristiques constitue un facteur de modulation très important de la croissance, de la morphologie et des caractéristiques d'échange de $\mathrm{CO}_{2}$ et de $\mathrm{H}_{2} \mathrm{O}$ des plants, tant en 
conditions nutritionnelles optimales qu'en conditions limitantes (Tableaux I, II et III). Ces résultats montrent que les différents composants associés à la tourbe ne doivent pas être considérés comme des simples produits de substitution ou de dilution, mais confèrent au produit des caractéristiques physico-chimiques bien tranchées.

Il n'est pas possible, dans le cadre de cette étude, d'établir de façon rigoureuse les liens de cause à effet entre les différentes grandeurs caractérisant soit l'ensemble de la période d'élevage (croissance, morphologie racinaire), soit uniquement l'état final (échanges gazeux, activité racinaire). II paraît toutefois logique, en raison de la nature du facteur étudié, de chercher à expliquer les performances de croissance des plants à l'aide des observations faites sur le développement racinaire.

Ainsi note-t-on de façon globale une opposition nette entre d'une part les substrats possédant une fraction volumique air (Fig. 1) importante (TA et TE) et caractérisés par un développement important du système racinaire et une croissance élevée des plants, et d'autre part des substrats à faible (TP, TEt) ou très faible (Tt) macroporosité n'autorisant qu'un développement racinaire et une croissance réduits. Cette opposition est toutefois à nuancer par la croissance médiocre des plants TE en 1986. Il est à noter que, malgré leur croissance médiocre, ces plants étaient caractérisés par un développement racinaire statisfaisant (Tableau II). Cette observation est bien en accord avec les propriétés physiques favorables du substrat (Fig. 1); elle permet également d'écarter, selon toute vraisemblance, l'hypothèse d'un effet de phytotoxicité (Moinereau et al., 1987) de l'écorce utilisée en 1986. De tels effets se caractérisent en effet par une forte inhibition du développement racinaire. La faible croissance des plants TE (et également des plants TEt) en conditions nutritionnelles limitantes est probablement due à une déficience azotée induite par la poursuite des processus de minéralisation de l'écorce.

Malgré les caractéristiques très favorables à la croissance du substrat $T A$, il importe de noter que ce substrat possède une réserve d'eau faible entre $\mathrm{pF} 1$ et $\mathrm{pF} 2$, augmentant les risques d'occurrence de situations d'alimentation hydrique limitante et nécessitant un contrôle très strict des disponibilités hydriques (Nicolas, 1986; Rivière et Nicolas, 1987). II est très probable que les performances très faibles des plants HP en 1985 soient liées à un défaut d'alimentation hydrique des plants, la réserve hydrique facilement disponible de ce substrat, malheureusement non déterminée ici, étant certainement sensiblement plus faible encore que celle de TA.

En 1985 et de façon plus sensible en 1986, la croissance des plants TP était inférieure à celle des plants TA. Cet effet dépressif apparaît comme étant lié à un développement racinaire et une densité de racines latérales courtes réduits. Ce faible développement racinaire est à mettre en relation d'une part avec la faible importance de la fraction air caractérisant le substrat TP (Fig. 1) et d'autre part avec les interactions physiques, décrites ci-dessus, entre les apex racinaires et les éléments de pouzzolane.

La nature du substrat affecte aussi de façon très sensible les caractéristiques photosynthétiques et transpiratoires des plants observées en fin de période d'élevage. Les effets du substrat sur la densité de flux de photosynthèse sont indépendants des effets affectant la surface foliaire (Tableau III, Fig. 1). En conditions de fertilisation optimale, on note une relation linéaire passant par l'origine entre les valeurs de $A$ et $g_{s}$ (Fig. 4). Cette relation 
linéaire indique que la variabilité individuelle globale et inter-traitements est liée en premier à une variabilité de la capacité photosynthétique mésophyllienne (Gueht, 1985) probablement induite en partie par des différences d'état nutritionnel des plants (Wong et al., 1985). Cette relation traduit également un couplage efficient entre $A$ et $g_{s}$ assurant une constance relative du coût en eau pour l'assimilation de $\mathrm{CO}_{2}$ (Tableau III). En conditions de fertilisation limitante on note un couplage moins efficient entre $A$ et $g_{s}$ traduisant d'une part un coût en eau global plus élevé et une discrimination entre substrats concernant ce critère (Tableau III).

Les caractéristiques d'assimilation de $\mathrm{CO}_{2}$ observées en fin de période d'élevage ne sont liées que de façon très lâche à la croissance pondérale des plants, et probablement uniquement par la corrélation globale liant le poids des plants à leur surface foliaire (Fig. 3). Cette absence de relation étroite pourrait être liée d'une part à l'existence de vitesses de mise en place des surfaces foliaires différentes entre individus, et d'autre part à une modification dans le temps du contrôle interne du niveau de $\mathrm{A}$.

Le résultat le plus significatif de la présente étude est constitué par la corrélation très étroite existant entre les valeurs moyennes par traitement de la capacité photosynthétique totale des plants et leur reprise après plantation (Fig. 5). Cette relation suggère, pour les plants transplantés avec motte intacte, une relation de cause à effet entre la capacité totale instantanée de production d'assimilats des plants et leur potentiel de croissance racinaire. La confirmation d'une telle relation pourrait être apportée par l'étude des relations entre photosynthèse et croissance racinaire au niveau individuel. En tout état de cause, l'établissement de la capacité photosynthétique des plants apparaît, du moins dans le cas de plants transplantés avec motte intacte, comme un excellent critère de qualité des plants vis-à-vis de la reprise après plantation. II est à noter que, dans la présente étude, la reprise des plants est liée de façon négative au poids relatif des racines par rapport à la partie aérienne souvent utilisée dans la pratique comme indicateur de la qualité des plants. Cette observation montre bien les limites de l'utilisation de critères morphologiques et visuels pour évaluer la qualité des plants et fait ressortir la nécessité de la mise au point de critères physiologiques plus fiables et qui puissent être facilement transférés dans la pratique (Aussenac et al., 1988).

Enfin, les résultats obtenus dans la présente étude montrent l'intérêt de l'utilisation en pépinière de certains substrats de culture artificiels (TA et TE dans notre cas) ayant des propriétés physico-chimiques plus favorables à la croissance, et entraînant une reprise après plantation meilleure, que les substrats classiques comprenant une proportion importante de terre.

\section{Remerciements}

Cette étude a été en partie financée par la Chambre Régionale d'Agriculture de Languedoc-Roussillon. Les auteurs tiennent à remercier L.M. Rivière, professeur à l'Ecole Nationale d'Ingénieurs des Travaux Horticoles à Angers, pour la communication des données relatives aux caractéristiques hydriques des substrats, et J.M. Ottorini, chercheur à la station de Sylviculture et Production à Nancy, pour ses conseils dans le traitement statistique des données. $\mathrm{Ce}$ travail a été rendu possible grâce à la collaboration technique de B. Clerc, J.M. Desjeunes, P. Gross et F. Willm du Laboratoire de Bioclimatologie-Ecophysiologie de Nancy.

\section{Références}

André J.P. (1987) Propriétés chimiques des substrats. In : Les Cultures Hors Sol (D. Blanc, ed.), INRA, Paris, pp. 127-147 
Aussenac G., Guehl J.M., Kaushal P., Granier A. \& Grieu P. (1988) Critères physiologiques pour l'évaluation de la qualité des plants forestiers avant plantation. Rev. For. Fr. 40s, 131 139

Caemmerer S. \& Farquhar G.D. (1981) Some relationships between the biochemestry of photosynthesis and the gas exchange of leaves. Planta 153, 376-387

Garbaye J. (1986) La production rapide de plants feuillus sur tourbe fertilisée. Les bases de la technique. Rev. For. Fr. 38 (3), 213-219

Garbaye J. \& Le Tacon F. (1978) Production de plants de chêne et de hêtre sur tourbe fertilisée. Rev. For. Fr. 30 (6), 445-452

Gras R. (1987) Propriétés physiques des substrats. In : Cultures Hors Sol (D. Blanc, ed.), INRA, Paris, pp. 79-126

Guehl J.M. (1985) Optimisation des relations entre transpiration et photosynthèse. Observations à propos des méthodologies d'étude. Ann. Sci. For. 42 (4), 397-410

Lemaire F., Dartigues A. \& Rivière L.M. (1980) Properties of substrates with ground pine bark. Acta Hortic. 99, 67-80

Moinereau J., Herrmann P., Favrot J.C. \& Rivière L.M. (1987) Les substrats. Inventaire, carac- téristiques, ressources. In : Les Cultures Hors Sol (D. Blanc, ed.), INRA, Paris, pp. 15-77

Nicolas H. (1986) Transferts hydriques dans le système substrat-plante-atmosphère. Application au pilotage automatique de lirrigation des cultures en conteneurs. Thèse de Docteur-Ingénieur, Ecole Nationale Supérieure Agronomique de Rennes

Riedacker A. (1978a) Premiers essais d'élevage de plants de chêne et de hêtre sur tourbe et sous tunnel plastique. Rev. For. Fr. 30 (6), 453458

Riedacker A. (1978b) Etude de la déviation des racines horizontales ou obliques de boutures de peuplier qui rencontrent un obstacle : application à la conception de conteneurs. Ann. Sci. For. 35 (1), 1-18

Rivière L.M. \& Nicolas H. (1987) Conduite de l'irrigation des cultures hors sol sur substrats. Contraintes liées au choix des substrats. Bull. GFHN 22, 47-70

Wong S.C., Cowan I.R. \& Farquhar G.D. (1985) Leaf conductance in relation to rate of $\mathrm{CO}_{2}$ assimilation. I. Influence of nitrogen nutrition, phosphorus nutrition, photon flux density and ambient partial pressure of $\mathrm{CO}_{2}$ during ontogeny. Plant Physiol. 78, 826-829 